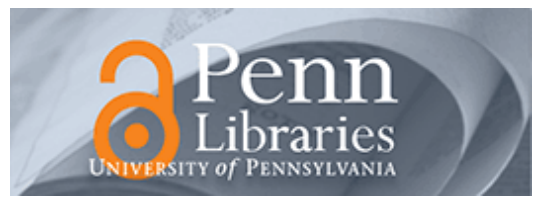

University of Pennsylvania ScholarlyCommons

Marketing Papers

Wharton Faculty Research

2001

\title{
Potential Diffusion of Expert Systems in Forecasting
}

\author{
J. Scott Armstrong \\ University of Pennsylvania, armstrong@wharton.upenn.edu \\ J. Thomas Yokum \\ Angelo State University
}

Follow this and additional works at: https://repository.upenn.edu/marketing_papers

Part of the Marketing Commons

\section{Recommended Citation}

Armstrong, J. S., \& Yokum, J. T. (2001). Potential Diffusion of Expert Systems in Forecasting. Retrieved from https://repository.upenn.edu/marketing_papers/144

Suggested Citation:

Armstrong, J.S. and Yokum, J.T. (2001). Potential Diffusion of Expert Systems in Forecasting. Technological Forecasting and Social Change. Vol. 67(1). p. 93-103.

The published version of this article is available at http://www.sciencedirect.com/science/article/pii/ S0040162599000955

This paper is posted at ScholarlyCommons. https://repository.upenn.edu/marketing_papers/144

For more information, please contact repository@pobox.upenn.edu. 


\title{
Potential Diffusion of Expert Systems in Forecasting
}

\author{
Abstract \\ We drew upon findings from the diffusion literature to assess the prospects for the diffusion of expert \\ systems in forecasting. Forecasters judged potential adoption of expert systems in relation to two \\ techniques that had been widely adopted in the past, Box-Jenkins and scenarios. They also rated each \\ technique on seven innovation characteristics: relative advantage, compatibility, divisibility, \\ communicability, complexity, product risks, and psychological risks. The respondents were classified by \\ four forecaster roles: researcher, educator, practitioner, and decision maker. In general, the expected \\ probabilities of adoption for expert systems were slightly higher than for the two other techniques. \\ Additionally, the respondents rated expert systems nearly equivalent to Box-Jenkins and scenarios on \\ relative advantage and communicability. In relating the probabilities of adoption to the characteristic \\ ratings, the groups perceived significant negative psychological and product risks with expert systems. \\ However the experts, especially practitioners and decision makers, rated expert systems positive on \\ compatibility, divisibility, and communicability, so it may be desirable to ensure that these positive traits \\ are stressed with potential adopters, especially researchers and educators. ( 2001 Elsevier Science Inc

\section{Disciplines} \\ Business | Marketing

\section{Comments} \\ Suggested Citation: \\ Armstrong, J.S. and Yokum, J.T. (2001). Potential Diffusion of Expert Systems in Forecasting. \\ Technological Forecasting and Social Change. Vol. 67(1). p. 93-103. \\ The published version of this article is available at http://www.sciencedirect.com/science/article/pii/ \\ s0040162599000955
}


Published in Technological Forecasting and Social Change, 67, 2001, 93-103.

\title{
Potential Diffusion of Expert Systems in Forecasting
}

\author{
J. Scott Armstrong \\ The Wharton School, University of Pennsylvania
}

Thomas Yokum

Angelo State University

\begin{abstract}
We drew upon findings from the diffusion literature to assess the prospects for the diffusion of expert systems in forecasting. Forecasters judged potential adoption of expert systems in relation to two techniques that had been widely adopted in the past, Box-Jenkins and scenarios. They also rated each technique on seven innovation characteristics: relative advantage, compatibility, divisibility, communicability, complexity, product risks, and psychological risks. The respondents were classified by four forecaster roles: researcher, educator, practitioner, and decision maker. In general, the expected probabilities of adoption for expert systems were slightly higher than for the two other techniques. Additionally, the respondents rated expert systems nearly equivalent to Box-Jenkins and scenarios on relative advantage and communicability. In relating the probabilities of adoption to the characteristic ratings, the groups perceived significant negative psychological and product risks with expert systems. However the experts, especially practitioners and decision makers, rated expert systems positive on compatibility, divisibility, and communicability, so it may be desirable to ensure that these positive traits are stressed with potential adopters, especially researchers and educators. (C) 2001 Elsevier Science Inc.
\end{abstract}

\section{Introduction}

We drew upon findings from the diffusion literature to assess the prospects for the diffusion of expert systems in forecasting [1-10].

Expert systems offer promise because they can combine the expertise of forecasters concerning techniques and data with expert knowledge about the situation to be forecast. This allows managers to use their knowledge in a formal way when making sales forecasts. Thus, in the case of General Motors automobile sales, the experts could make adjustments for the effect of a recent strike. But despite its apparent advantages, and despite some initial validation studies demonstrating improved accuracy, there has been no rush by managers to adopt expert systems for sales forecasting.

This article investigates factors that encourage or discourage the adoption of expert systems for forecasting. This could lead to advice on how to implement expert systems more effectively. To provide benchmarks for our study, we looked at two techniques that have been widely adopted: Box-Jenkins and scenarios. How do the prospects for adoption of expert systems compare with those for these more established techniques?

Box-Jenkins has been adopted by many academics since 1970. It has also been used by practitioners [1]. Dalrymple [2] found that many practitioners and decision makers were dissatisfied with it, but Mentzer and Kahn [3] found somewhat higher satisfaction. While Box-Jenkins does not produce more accurate forecasts than 
alternative time series procedures, it seems likely that it forecasts at least as accurate as those produced judgmentally, and they are far less expensive. The adoption of this procedure is aided by the availability of programs that will automatically make most of the statistical decisions.

Scenarios are primarily useful as a tool for gaining acceptance of forecasts. As we use the term, scenarios involve writing stores about forecasts so that decision makers will take them seriously. Research by psychologists has demonstrated numerous aspects that will increase the perceived likelihood of a forecast [4]. This is particularly useful when dealing with forecasts that have unpleasant consequences. The term "scenarios" has been loosely used, often as a substitute for the term "alternatives." Thus, while it has been widely accepted by decision makers, its use as a way to gain acceptance is probably limited.

We relied on diffusion theory to identify the factors related to the adoption of new products and the next section describes how it applies to forecasting techniques. This is not a test of diffusion theory. Rather, it is an attempt to determine whether previous findings apply to forecasting, and, if so, what are the implications for gaining adoption of expert systems.

Our basic approach was to obtain experts' opinions about the prospects for adoption of expert systems. In doing so, we were interested in whether opinions might vary by the role of the adopter. We solicited the opinions of researchers, educators, practitioners, and decision makers.

\section{Design of the Study}

Diffusion, as we use the term, is the process by which innovations are disseminated among members of a market. We applied a simple diffusion framework first proposed by Rogers in 1962. He attributes the adoption of innovations to seven characteristics: (1) relative advantage involves whether the proposed technique is superior to the current procedure (for example, a new forecasting technique might be more accurate); (2) compatibility refers to whether the technique fits in with the prior experiences and existing behavior of the potential adopters; (3) divisibility is the degree to which the adopter can experiment with the technique; (4) communicability is the case with which the technique can be described to potential users; (5) complexity is the extent to which the technique is difficult to understand or use; (6) product risks relate to the use of the product (e.g., whether the technique may break down in critical situations); and (7) psychological risks represent the reactions (real or perceived) of peer groups. These characteristics have been well-supported by diffusion research on new product, ideas, or techniques [5].

\section{Expert Opinions Procedures}

We studied the opinions of forecasting experts selected from a list provided by the International Institute of Forecasting (1117). This list included members and nonmembers of the 1117. There were 322 responses from the 738 names in the mail-out sample.

We solicited opinions from experts who had different roles. The four forecaster roles were researcher, educator, practitioner, and decision maker. Generally, researchers are the inventors who create, develop, and test new techniques, so they should be concerned about such things as demonstrating relative advantage. Educators communicate information about the new techniques. Practitioners implement the forecasting methods and make forecasts so they are expected to be interested in compatibility. Decision makers use the forecasts to make decisions so they should be interested in compatibility and relative advantage.

To identify group membership, we asked the respondents to allocate 100 points among the four forecasting roles. Group membership was determined by the respondent's primary allocation of points to the four categories. Of the respondents, 94 allocated more points to the researcher role, 58 categorized themselves as educators, 132 as practitioners, and 38 as decision makers. Definitions for each group, as presented to survey respondents, are provided in Appendix A. 
Experts were provided with descriptions of the forecasting techniques (as shown in Appendix B). The definitions are stated in simple terms and they ignore the details about the process. For example, we described expert system as being based on rules of experts whereas, in fact, they often rely upon findings from empirical research. We were interested in their opinions about any aspects of the techniques.

The probability of potential adoption (the dependent variable) for each technique was assessed by responses to a 100 point scale. The following question was asked of all those who were not presently using the specified technique, "What is the probability that you would adopt or use (specified technique) in the near future?" Present users were scored as $100 \%$.

We also asked the experts to rate the forecasting techniques according to each of the seven diffusion characteristics by using a seven-point agreement-disagreement scale. The wording for these characteristics is provided in Appendix C. Given the extensive research on diffusion and innovations [5], we expected that the forecast experts' perceptions about the three techniques would conform to Rogers' findings regarding adoption. Specifically, we expected relative advantage, compatibility, divisibility, and communicability to be positively related to the probability of adoption, while complexity, product risks, and psychological risks would be negatively related. Negative relationships for the seven factors or insignificant results for the first four would indicate perceived barriers for the diffusion of a forecasting technique.

\section{Results}

This section reports on a separate analysis of both the adoption probabilities and the innovation characteristics. This is followed by an analysis of the relationships between the probability of adoption and the innovation characteristics for the three techniques.

\section{Potential Adoption}

Although we did not find published evidence of any forecasting applications of expert systems at the time of our survey, some software packages for forecasting had been advertising that they contained expert systems. Thus, it is not surprising that a number of respondents replied that they had adopted expert systems. The unweighted average probability of adoption for expert systems was $33.4 \%$ for those who identified themselves as nonusers or past users. In rough terms, this compares favorably with Box-Jenkins at $25.4 \%$ and for scenarios at $30.2 \%$.

The forecast groups were similar in their stated probability of adoption for each of the techniques. Table 1 displays the results for the potential adoption for nonusers of the three techniques.

Table 1. Self-Assessed Probabilities of Adoption for the Three Forecasting Techniques (Number of Experts in Parentheses)

\begin{tabular}{lccc}
\hline \multirow{2}{*}{ Role } & \multicolumn{3}{c}{ Forecasting Techniques } \\
\cline { 2 - 4 } & Box-Jenkins & Scenarios & Expert Systems \\
\hline Researchers & $22.9(37)$ & $27.8(58)$ & $31.9(73)$ \\
Educators & $28.1(25)$ & $27.7(37)$ & $34.2(45)$ \\
Practitioners & $28.0(85)$ & $31.6(68)$ & $31.0(104)$ \\
Decision Makers & $22.5(23)$ & $33.7(18)$ & $36.3(27)$ \\
\hline Average & 25.4 & 30.2 & 33.4 \\
\hline
\end{tabular}

Ninety-five percent confidence intervals ( $95 \%$ CIs) were calculated for each of the adoption probabilities in Table 1. The average interval was \pm .14 , ranging from .09 for practitioners adopting expert systems to .22 for decision makers adopting scenarios. The confidence intervals all overlapped with one another, suggesting that there was little difference in potential adoption across techniques and groups. 
The implications of these findings are favorable for expert systems in that the expected adoption was equal to that for two of the more well-established techniques. It is also favorable in that prospects for adoption seem relatively consistent across each group of respondents. Finally, the prospects for adoption of expert systems were highest among decision makers, and this seems promising given that the other two techniques achieved relatively wide adoption.

\section{Innovation Characteristics}

Average ratings for the seven innovation characteristics (i.e., relative advantage, compatibility, divisibility, communicability, complexity, product risks, and psychological risks) for expert systems are listed in Table 2 . The ratings are seven-point agreement disagreement scores for diffusion statements. The innovation statements for the four positive characteristics are coded so that higher ratings indicate that the respondents' ratings of the statement connoted higher agreement with the innovation characteristic. The three negative characteristics (compatibility and the two product risk items) were reverse coded so that higher scores indicate less agreement with the statements. They are listed in order of agreement, and the ratings for the other two techniques are also provided.

Table 2. Average Innovation Characteristic Ratings by Forecasting Technique

\begin{tabular}{lccc}
\hline \multicolumn{1}{c}{$\begin{array}{c}\text { Innovation } \\
\text { Characteristic }\end{array}$} & Box-Jenkins & Scenarios & Expert Systems \\
\hline Complexity & $5.14(1.86)$ & $5.05(1.56)$ & $4.99(1.56)$ \\
Communicability & $4.48(2.02)$ & $4.93(1.45)$ & $4.73(1.65)$ \\
Product Risks & $4.29(1.76)$ & $4.39(1.49)$ & $4.24(1.62)$ \\
Psychological Risks & $4.28(1.60)$ & $4.18(1.39)$ & $4.24(1.38)$ \\
Relative Advantage & $3.37(1.65)$ & $4.40(1.47)$ & $4.19(1.71)$ \\
Divisibility & $3.95(1.67)$ & $4.01(1.38)$ & $3.80(1.54)$ \\
Compatibility & $3.74(1.82)$ & $3.65(1.45)$ & $3.12(1.39)$ \\
\hline
\end{tabular}

Ratings are mean (Standard Deviations). Characteristic scales are measured from 7 as "Agree Strongly" to 1 as "Disagree Strongly."

The characteristic with the highest rating associated with expert systems was complexity (at 4.99). The average agreement with the statement, "I would have no difficulty in understanding how to use ..." was also high for BoxJenkins (5.14) and for scenarios (5.05). These averages can be interpreted to mean that respondents were not concerned with problems of technique complexity. (We suspect that the respondents are overly optimistic with respect to their ability to understand Box-Jenkins). Communicability was the next most highly rated characteristic for expert system: "I think if I used ..., I would have no difficulty in telling my colleagues what they are." This was followed by product risks and psychological risks. There was less concern about failure of the techniques than about peer reactions.

Relative advantage (“... would offer real advantages over the way I presently do things") was only the fifth most favorably rated characteristic for expert systems. This was followed by divisibility, "1 wouldn't have to spend much time with ... in order to try out the idea." The lowest rated characteristic was compatibility: "If I used ..., I would have to change some of the ways I do things."

The rank order of the characteristic agreement scores was generally the same across techniques (see Table 2). The biggest difference in the rank order of the characteristics was for relative advantage, which was fifth for expert systems, seventh for Box-Jenkins, and sixth for scenarios.

Standard deviations of the experts' ratings summarize the extent of agreement. Standard deviations for the characteristics were generally similar for the characteristics of expert systems (ranging from 1.38 to 1.71 and averaging 1.55). However, the highest dispersion measures were for Box-Jenkins (1.77), followed by expert 
systems, then scenarios (1.46). Thus, despite its newness, the forecasting experts were generally in as much agreement about the characteristics of expert systems as for the other methods.

These innovation characteristic ratings were also analyzed by forecasting role. Few significant differences occurred among roles. That is, the ratings for the seven Rogers' characteristics seem to apply roughly the same, irrespective of one's role in forecasting.

\section{Innovation Characteristics Related to the Probability of Adoption}

Table 3 shows the results for examining the consistency of Rogers' framework as applied to the three techniques. The relationship between the probability of adoption and the seven innovation characteristics for expert systems and for the two bench mark techniques were estimated by logistic regression. The coefficients (intercepts omitted) represent the forecasting experts' perceptions of the average increase or decrease in the log odds of the adoption probability given a. one-point increase in the agreement/disagreement scale for each of the seven attributes. The chi-square $\left(\mathrm{X}^{\prime}\right)$ likelihood ratios, equivalent to an F-test on OLS coefficients that the vector of coefficients are zero, were all significant at the .05 level. The magnitude of the logistic coefficient gives the relative importance of the dimension,, while the sign of the coefficient indicate whether the dimension aids (positive) or harms (negative) adoption. For example, the .487 coefficient for Expert Systems' relative advantage can be interpreted to mean that there is a .487 increase in the log odds of adopting Expert Systems for each marginal increase of one in its relative advantage rating, or equivalently .221 increase (i.e., $\mathrm{e}^{487}$ ) in the odds of adoption. This analysis suggests the best way to promote the adoption of expert systems is by improving its real or perceived relative advantage. Also, important for expert systems is the reduce its significantly negative characteristics (i.e., complexity, product and psychological risks) and to make improvements in its nonsignificant positive characteristics (i.e., compatibility, divisibility, and communicability).

Table 3. Relationship between Diffusion Characteristics and Self-Assessed Probabilities of Adoption-Logit Coefficients

\begin{tabular}{|c|c|c|c|c|c|c|c|c|}
\hline & $\begin{array}{c}\text { Relative } \\
\text { Advantage }\end{array}$ & Compatibility & Divisibility & $\begin{array}{c}\text { Communic } \\
\text {-ability }\end{array}$ & Complexity & $\begin{array}{l}\text { Product } \\
\text { Risks }\end{array}$ & $\begin{array}{c}\text { Psychological } \\
\text { Risks }\end{array}$ & $\begin{array}{c}\text { Chi-Square } \\
\text { Likelihood } \\
\text { Ratio } \\
\end{array}$ \\
\hline Box-Jenkins & $0.458^{*}$ & $0.414^{*}$ & -0.078 & -0.075 & $-0.262^{*}$ & $-0.387^{\star}$ & $-0.198^{*}$ & $144.845^{\star}$ \\
\hline Scenarios & $0.630^{*}$ & $0.327^{*}$ & -0.160 & -0.059 & $-0.238^{*}$ & -0.139 & $-0.575^{\star}$ & $124.863^{*}$ \\
\hline Expert Systems & $0.487^{\star}$ & 0.132 & 0.025 & 0.135 & -0.135 & $-0.185^{*}$ & $-0.399^{*}$ & $122.765^{\star}$ \\
\hline
\end{tabular}

${ }^{*} p<0.05$, one-tail test. Note: Bold coefficients represented unexpected $+/-$ results.

The signs of the coefficients agreed, at least for expert systems, with Rogers' framework as to why the forecasting experts would adopt a method. Positive coefficients were found for relative advantage, compatibility, divisibility, and communicability, with only the first significant. Significant negative coefficients were obtained for all expected negative coefficients including complexity and product risk and psychological risks. The four bolded coefficients in Table 3 show where the expected positive sign was not obtained for Box-Jenkins and Scenarios techniques; none of these was statistically significant.

There might be some reason to expect differences between the response coefficients for the different techniques. For example, the complexity of Box-Jenkins may cause the coefficient of log odds to be more negative in relation to the probability of adoption than other techniques. Likewise, the communicability of Box-Jenkins would seem to be low for the same reason. $F$-tests for homogeneity were performed on the innovations (Johnston 1972). While this test is usually performed in relation to OLS coefficients, the same problems are prevalent with logistic regression. On the three innovations, F-tests for pooled slopes, intercepts, and overall homogeneity were rejected $(\mathrm{F}=1.96, p<.05 ; F=14.02, p<.01 ; F=3.65 p<.01)$. Even with the results indicating differences, we basically obtained consistent coefficient values across the three techniques (Table 3). However, the separate regressions still showed differences on the same relationships. 


\section{Innovation Characteristics Related to the Probability of Adoption by Forecast Role}

The 12 logistic regressions in Table 4 portray the effects of the innovation characteristics on the four expert groups. The suggested relationships generally explained how much of the cross-sectional data varied. The chi-square likelihood ratio were again all significant a the .05 level. Conditional indices (2) for the twelve relationships indicated little problem with multicollinearity, which is consistent with the assumption that the coefficients represent independent dimensions. The amount of uncertainty (standard errors of each characteristic) varied across the four groups. The standard errors for the practitioners were the lowest, approximately one-half as large as for decision makers, while for educators and researchers they were in the middle of the other two groups. This means there was more of a difference of opinions among decision makers with regard to their ratings.

Table 4. Relationship between Diffusion Characteristics and Probabilities of Adoption: Logistic Coefficients by Role

\begin{tabular}{|c|c|c|c|c|c|c|c|c|}
\hline & $\begin{array}{c}\text { Relative } \\
\text { Advantage } \\
\end{array}$ & Compatibility & Divisibility & $\begin{array}{c}\text { Communic } \\
\text {-ability }\end{array}$ & Complexity & $\begin{array}{c}\text { Product } \\
\text { Risks }\end{array}$ & $\begin{array}{c}\text { Psychological } \\
\text { Risks } \\
\end{array}$ & $\begin{array}{c}\text { Chi-Square } \\
\text { Likelihood } \\
\text { Ratio } \\
\end{array}$ \\
\hline \multicolumn{9}{|l|}{ Box-Jenkins } \\
\hline Researcher & $0.697^{*}$ & 0.214 & -0.198 & 0.207 & -0.068 & $-0.508^{*}$ & -0.040 & $54.48^{*}$ \\
\hline Educator & 0.333 & $0.831^{*}$ & -0.461 & $-0.870^{*}$ & -0.097 & $-0.898^{*}$ & $-0.788^{*}$ & $42.86^{*}$ \\
\hline Practitioner & $0.527^{*}$ & $0.444^{*}$ & 0.068 & -0.186 & $-0.271^{*}$ & $-0.166^{*}$ & $-0.420^{*}$ & $61.06^{*}$ \\
\hline Decision-maker & $0.879^{*}$ & 0.387 & $-1.01^{*}$ & 0.781 & -0.110 & $-1.32^{*}$ & 0.381 & $70.38^{*}$ \\
\hline \multicolumn{9}{|l|}{ Scenarios } \\
\hline Researcher & $0.525^{*}$ & 0.099 & -0.094 & 0.104 & -0.243 & -0.014 & $-0.682^{*}$ & $33.75^{*}$ \\
\hline Educator & $0.750^{*}$ & $0.957^{*}$ & -0.072 & -0.365 & -0.157 & -0.197 & -0.882 & $30.52^{*}$ \\
\hline Practitioner & $1.01^{*}$ & $0.379^{*}$ & -0.251 & -0.207 & $-0.585^{\star}$ & 0.047 & $-0.840^{*}$ & $83.19^{*}$ \\
\hline Decision-maker & 0.856 & $2.056^{*}$ & -0.206 & 0.203 & 0.402 & 0.131 & -0.289 & $15.69^{*}$ \\
\hline \multicolumn{9}{|l|}{ Expert Systems } \\
\hline Researcher & $0.567^{*}$ & 0.039 & 0.081 & -0.035 & -0.222 & -0.235 & $-0.615^{*}$ & $49.25^{*}$ \\
\hline Educator & 0.051 & -0.258 & -0.257 & 0.129 & -0.153 & -0.396 & $-0.743^{*}$ & $22.15^{*}$ \\
\hline Practitioner & $0.481^{*}$ & $0.286^{*}$ & 0.132 & 0.116 & -0.123 & -0.188 & $-0.430^{*}$ & $57.36^{*}$ \\
\hline Decision-maker & 0.555 & $0.854^{*}$ & -0.375 & 0.301 & -0.487 & 0.105 & -0.240 & $15.06^{*}$ \\
\hline
\end{tabular}

${ }^{*} p<0.05$, one-tail test. Note: Bold coefficients represented unexpected $+/-$ results.

F-tests for homogeneity were also performed in relation to the four groups. The results, opposite from the last section, indicated that there was no need to reject, in each case, the null hypotheses of homogeneity of the groups (i.e., all $9 F \mathrm{~s}, p>.05$ ).

Of the 84 logistic coefficients that represented the relationships between the log odds of adoption and the characteristic ratings of the four groups for the three techniques, $76 \%$ had the correct sign as suggested by the Rogers' framework [5] and 24\% of the coefficients had an incorrect sign (appear in bold in Table 4). Thirty-five percent of all coefficients were significant (Wald chi-square test, $p<.05$, one-tail tests), but only two of these had effects opposite to expectations.

None of the four role groups displayed significant negative effects for experts system's product risks in relation to the log odds of adoption. However, all three techniques showed some role significant reduction in log odds adoption for psychological risks, the fear of peer disapproval. This was not basically true for decision makers.

Practitioners responded the most favorably toward expert systems. The expert systems' logistic regression coefficients were significant and positive for relative advantage (.481) and compatibility (.286), but they were significant and negative for psychological risks (-.430). All their ratings were equivalent to their ratings on the other two bench mark techniques. Researchers produced significant positive ratings for relative advantage (.567), also equivalent to their ratings on the benchmarks. The two academic groups also produced significantly negative coefficients for psychological risks (-.615, -.743). None of the other logistic coefficients for the other dimensions 
were either significantly positive (compatibility, divisibility, communicability) or significantly negative (complexity, product risks) for researchers or educators. With the exception of compatibility the same was true for practitioners. Decision makers produced only one significantly positive rating, that for the compatibility dimension (.854). In comparing the ratings of expert systems to the bench marks, the patterns of significantly positive ratings were mixed and sparse for all three methods.

The only exception was the significant negative ratings for Box-Jenkins' product risks, whereas there was none for expert systems.

We used the probability of adoption as the dependent variable in the relationship to the innovation characteristics. This is an intention measure, not actual adoption. While intentions provide useful predictions of behavior for important decisions, the correspondence between intentions and behavior is not strong. The biases inherent in his measure are expected to be common across groups and across techniques in our analysis.

The sample size was not large when one considers that the analyses were done for each of the four roles. In particular, there were only 38 decision makers. This is reflected in the high standard errors.

\section{Discussion}

Those who plan to implement expert systems might use Rogers' framework as a guide when they consider how various groups might respond. Our results, on using perceived adoption, are consistent with Rogers' framework. The results in Table 4 suggest which characteristics of this framework are most important to the adoption by each of the groups. For example, decision makers were especially interested in the compatibility of expert systems. To gain adoption then, it might help to tailor expert systems so that they represent the policies of the experts who are using the forecasts.

Relative advantage was important for the adoption of expert systems. The accuracy of expert systems could be compared with the accuracy of the method currently used to make forecasts. Collopy et al. [6] presents guidelines for making such an evaluation. Other criteria such as ease of use, ease of interpretation, and cost savings, may also enhance the perception of relative advantage.

Interestingly, although the perceived relative advantage of expert systems was similar to that for scenarios, its rating was not high in absolute terms (4.19 of 7). The evidence in favor of expert systems is modest [7]. Furthermore, it is unlikely that our respondents were aware of the evidence.

One question in our survey revealed that respondents had a favorable view toward expert systems when a specific application was considered. Strong agreement was obtained for the statement that "Rules derived from experts could, if properly coded and applied by a computer program, improve the accuracy of forecasting by more effectively selecting among available extrapolation methods." Seventy percent of the 319 respondents agreed and only 12 percent disagreed. There was agreement across all four groups on this item.

Researchers should focus on using empirical findings in the development of expert systems. In contrast, practitioners should focus initially on simple expert systems and should draw upon the expertise of those who are currently making forecasts. Once simple expert systems have been implemented, further refinements can bring in the empirical findings about forecasting methods, drawn from academic research.

At the start of our study, we had taken it for granted that expert systems would improve forecast accuracy. We found that our assumption was not based on strong evidence and that many experts did not agree with our assumption. Thus, it seems that researchers and practitioners should assess whether the use of the expert systems does provide a relative advantage. 


\section{Conclusions}

Prior research suggested that the analysis of general adoption characteristics helps to explain the adoption of products. We used these findings to examine characteristics related to the adoption of expert systems.

Expert systems were viewed favorably in comparison with two techniques that have been widely adopted. Practitioners, educators, and researchers tended to focus on the relative advantage of expert systems. The relative advantage of expert systems was rated as well above Box-Jenkins, although not quite as high as scenarios. Decision makers differed somewhat from the other three groups; while they were the most favorable towards the compatibility of expert systems, they saw problems with relative advantage. Their skepticism may be well-founded. There is a need for studies on the predictive ability of expert systems in comparison with other approaches and especially in comparison with the accuracy of existing procedures.

In comparison with two well-established forecasting techniques, expert systems appear to have reasonable prospects for diffusion, but some barriers are apparent. Psychological risks create barriers for expert systems, so this is a high risk area for academic research. Because of the problems with complexity merits attention, initial attempts at expert systems should be kept simple even if this involves some loss of predictive power.

\section{Acknowledgements}

The authors thank Fred Collopy, Jim Cox, Douglas Dalrymyple, Robert Fildes, Everette and Mary Ann Gardner, Donald Hughes, Lyman Ostlund, Joel Saegert, Daniel Sieden, and Tom Swamstrom for help with questionnaire development and comments on the research.

\section{References}

1. Mentzer, J. T., and Cox, J. (1984), "Familiarity, application, and performance of sales forecasting techniques," Journal of Forecasting, 3, 27-36.

2. Dalrymple, D. (1987), "Sales forecasting practices: Results from a United States survey," International Journal of Forecasting, 3, 379-391.

3. Mentzer, J. T., and Kahn, K. B. (1995), "Forecasting technique familiarity, satisfaction, usage, and application," Journal of Forecasting, 14, 465-476.

4. Gregory, W., and Duran, A. (2001), "Scenarios and acceptance of forecasts," in J. S. Armstrong, Principles of Forecasting: A Handbook for Researchers and Practitioner, Norwall, MA: Kluwer Academic Publishers, pp. 519-541.

5. Rogers, E. M. (1995) Diffusion of Innovations. New York: The Free Press.

6. Collopy, F., Adya, M., and Armstrong, J. S. (1994), "Principles for examining predictive validity: The case of information systems spending forecasts," Information Systems Research, 5, 170-179.

7. Collopy, F., Armstrong, J. S., and Adya, M., "Expert systems for forecasting," in J. S. Armstrong, Principles of Forecasting: A Handbook for Researchers and Practitioner, Norwall, MA: Kluwer Academic Publishers, pp. 285-300.

8. Armstrong, J. S. (1985), Long-Range Forecasting: From Crystal Ball to Computer, 2nd ed. New York: J. Wiley and Sons.

9. Belsley, D., Kuh, E., and Welsch, R. (1980), Regression Diagnostics: Identifying Influential Data and Sources of Collinearity. New York: J. Wiley and Sons. 
10. Collopy, F., and Armstrong, J. S. (1992), "Rule-based forecasting: Developing and validation of an expert systems approach to combining time series extrapolations, Management Science, 38, 1394-1414.

\section{APPENDIX A \\ Forecast Group Definitions}

Researchers - Individuals who primarily pursue the development and testing of forecasting theory. They disseminate knowledge through professional speeches and research articles

Educators - those who formally educate others about forecasting thought and theory. Dissemination of knowledge involves direct contact, usually within the environment of a university, but also through private firms, professional organizations, various seminars, professional meetings, and text books.

Practitioners - Those who help to prepare and present forecasts within a profit, non-profit, or public organization. Their knowledge about forecasting is based on their experience, procedures developed by the organization, and the efforts of educators and researchers.

Decision Makers - Individuals within a firm who use forecasts to plan, make decisions, implement strategies, and control performance measures. They depend upon the forecasting practitioner.

\section{APPENDIX B \\ Forecasting Techniques}

Expert Systems - Computer programs that use rules developed from the way experts think. They decide what forecasting method is appropriate in a given situation and how it should be used.

Box-Jenkins - Sophisticated statistical technique that estimates and projects the structure of time series data. It is a class of models called ARIMA (Autoregressive Integrated Moving Average). These forecasts use only historical data on the series being forecast.

Scenarios - Stories describing the environment, how a strategy can be implemented, and what results are likely. Scenarios have been proposed as a structured way to allow organizations to plan, given unusual or unpleasant forecasts.

\section{APPENDIX C Forecasting Characteristics}

Relative Advantage - “(Box-Jenkins, Scenarios, Expert Systems) would offer real advantages over the way I presently do things."

Compatibility - "If I used (Box-Jenkins, Scenarios, Expert Systems), I would have to change some of the ways I do things."

Divisibility - "I wouldn't have to spend much time with (Box-Jenkins, Scenarios, Expert Systems) in order to try out the idea."

Communicability - "I think if I used (Box-Jenkins, Scenarios, Expert Systems), I would have no difficulty in telling my colleagues what they are."

Complexity - "I have no difficulty in understanding how to use (Box-Jenkins, Scenarios, Expert Systems)." 
Product Risks - "If 1 used (Box-Jenkons, Scenarios, Expert Systems), the chances that I would be not satisfied with the way they performed are: ..." Scale was low (1) to high (7).

Psychological Risks - If 1 used (Box-Jenkins, Scenarios, Expert Systems), the chances that my colleagues would approve are:..." Scale was low (1) to high (7). 